\title{
A Wireless Sensor Network Model Using Douglas-Peucker Algorithm in The IoT Environment
}

\author{
Se-Jung Lim ${ }^{1}$ and Gwang-Jun Kim ${ }^{2 *}$ \\ ${ }^{1}$ Liberal Arts \& Convergence Studies, Honam University, 120, Honamdae-gil, \\ Gwangsan-gu, Gwangju-si, 62399, Republic of Korea \\ ${ }^{2}$ Department of Computer Engineering, Chonnam National University, 50, Daehak- \\ ro, Yeosu, Jeonnam, 59626, Republic of Korea \\ ${ }^{1}$ limsejung@honam.ac.kr, ${ }^{2} \mathrm{kgj} @ j n u . a c . k r$ (correspondingauthor)
}

\begin{abstract}
Wireless Sensor Networks (WSNs) which are the major component in the Internet of Things (IoT) mainly use interconnected intelligent wireless sensors. The operation of wireless sensors is typically powered by batteries and recharging batteries is difficult due to the rugged environments where they operate. Therefore, the study for prolonging the network lifetime is one of the most important challenges in WSNs. In this paper, we propose a Wireless Sensor Network Model (WSNM) using Douglas-Peucker algorithm in the IoT environment to prolong the network lifetime. The performance of WSNM is compared with PEGASIS and EnhancedPEGASIS. In the simulation results, the performance of WSNM outperforms PEGASIS and Enhanced-PEGASIS.
\end{abstract}

Keywords: Chain formation, Data collection, Douglas-Peucker algorithm, Internet of Things (IoT), PEGASIS, Wireless sensor networks (WSNs)

\section{Introduction}

Another foundational technology for the Internet of Things (IoT) is the Wireless Sensor Networks (WSNs), which mainly use interconnected intelligent sensors to sense and monitoring. WSNs are used for gathering data from their surrounding and delivering them to users and for accessing connected IoT devices remotely. They comprise of an extensive number of small nodes that can detect, compute, and communicate with other devices [1]. In fact, sensor nodes are generally powered by batteries, and it is difficult to add energy to the sensor nodes due to the rugged environments where they operate [2]. Therefore, the study of energy efficient routing algorithms is one of the most important challenges in WSNs. Many researchers have made an effort to prolong the network lifetime of WSNs [7][8][9][10][11][12][13][14][15]. Among them, Power-Efficient GAthering in Sensor Information Systems (PEGASIS) [8] is the most well-known routing algorithm based on a greedy chain formation approach in WSNs. Since PEGASIS-based routing algorithms using a greedy algorithm are gradually result in an increase of neighbor distances, some nodes consume much more energy to transmit data to their neighbors. In this paper, we propose a wireless sensor network model (WSNM) using DouglasPeucker algorithm in the IoT environment for solving this problem via the forming of balanced

Article history:

Received (June 25, 2019), Review Result (August 4, 2019), Accepted (October 16, 2019) 
chain. We conducted simulation experiments using OMNET++ and compare the performance of our algorithm with PEGASIS and Enhanced-PEGASIS [10]. To our knowledge, our study is the first paper that forms a balanced chain using Douglas-Peucker algorithm. The remainder of this paper is organized as follows. Related work is presented in section 2. We describe our algorithm in section 3 . The simulation results are described in section 4. Finally, we conclude this paper in section 5 .

\section{Related work}

\subsection{Pegasis}

In Power-Efficient GAthering in Sensor Information Systems (PEGASIS) [8], sensor nodes are randomly deployed in the network field and organized into chain using a greedy algorithm before the first round. The main idea of PEGASIS is that each node to receive from and transmit to close neighbors along a chain and take turns in being the leader for transmitting data to the BS. The farthest node from the BS is a starting node of the chain and the next node is selected using a greedy algorithm. If there is a sensor node already included in the chain, it is not able to revisit the sensor node. In each round, a leader node among sensor nodes gathers data along a chain from end nodes using the token passing mechanism. Each sensor node fuse its own data and data received from its neighbor in the chain and transmit the fused data toward to a leader node for onward transmission to the BS. The scheme of chain formation using a greedy algorithm in PEGASIS is shown in [Figure 1].

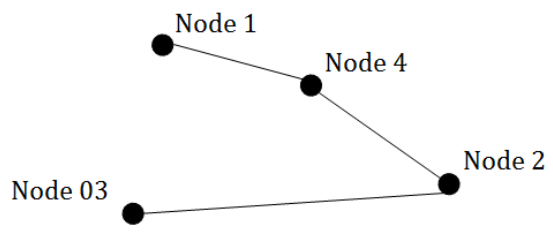

Figure 1. Chain formation using a greedy algorithm

\subsection{Douglas-Peucker algorithm}

Douglas-Peucker algorithm [3][4] is developed by Douglas and Peucker. This algorithm is the most commonly used global simplification algorithm in cartography and Geographic Information Systems (GIS).

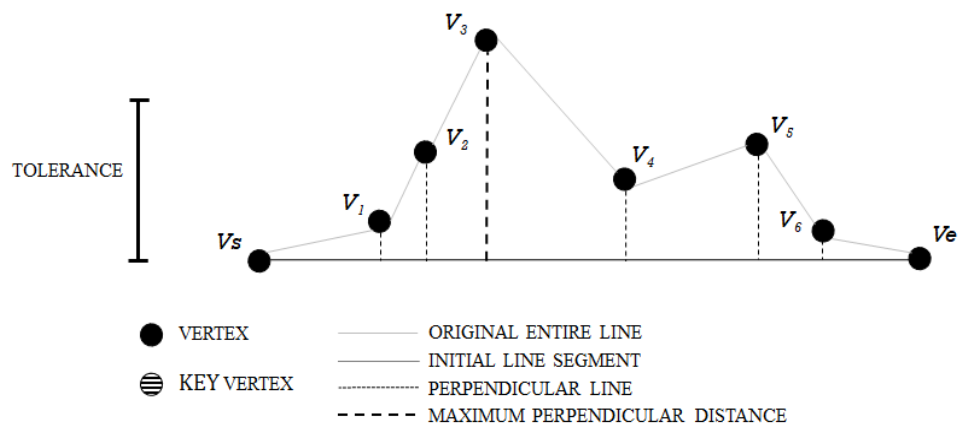

Figure 2. Process of line simplification 
As shown in [Figure 2], if $V s$ and $V e$ are respectively the start and end vertices in the original entire line, an initial line segment $\overline{V s V e}$ means the single chain which joining two vertices. The perpendicular distance from all intermediate vertices to that initial line segment $\overline{V s V e}$ is calculated. If the vertex has a maximum perpendicular distance from an initial line segment $\overline{V S V e}$ and is larger than a specified tolerance, this vertex is served as a key of the simplification in the next step. In other words, the initial line segment $\overline{V s V e}$ is segmented into two. This process is repeated until the perpendicular distance for all vertices of the entire original line is smaller than the specified tolerance. The intermediate vertex within the specified tolerance is eliminated and then the vertices that have the maximum perpendicular distance is connected as the result of simplification.

\section{Proposed algorithm}

\subsection{Chain formation phase}

The sink defines the node-to-edge distance tolerance $(\mathrm{T})$ based on the location of sensor nodes. In the network field, a single edge (SE) between a starting node (SN) the farthest from the sink and the farthest sensor node (EN: ending node) from the starting node is connected. The perpendicular distance of every intermediate node except for two nodes (SN and EN) from the connected SE is computed. If the maximum value among the computed perpendicular distance of intermediate nodes is larger than the defined node-to-edge distance tolerance (T), we set an intermediate node with this value to a perpendicular node (PN). The tentative edge (TE) from SN and EN to PN is respectively connected. This TE need to be redefined to the SE for the next segmentation of the network field. Until the maximum value of the perpendicular distance from intermediate nodes to the SE is smaller than the T, the above process is repeated. We start the balanced chain formation from the SN within the same segment using a greedy algorithm. The nearest PN is selected as the next node in case of all sensor nodes within one segment would be joined to the chain.

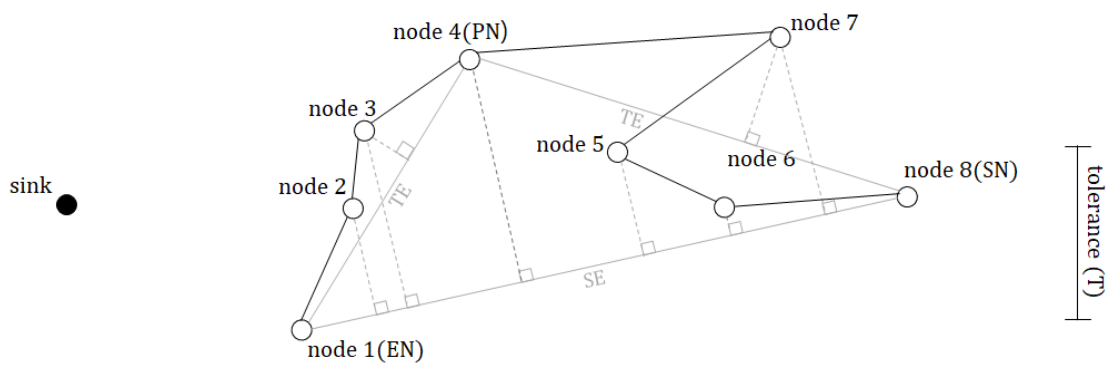

Figure 3. Formation of a balanced chain

\subsection{Data collection phase}

The data is collected toward the chain leader from the end nodes (SN and EN) along the chain by using the token passing mechanism. Since the token is very small, it does not affect the network lifetime. The sensor node fuses the data it receives from its neighbor with its own data and then transmits to the other neighbor on the balanced chain. The data fusion is performed at every sensor node except the SN and EN in the balanced chain. The chain leader commonly receives data from its both neighbors and transmits the fused data to the sink. In the 
chain, each sensor node communicates with its near neighbors to collect data and take turns being the chain leader.

\section{Simulation}

In this section, we evaluate the performance of our algorithm (WSNM) discussed in the previous section. Simulations are driven by OMNET++ [5][6] simulator and run on windows7. We firstly define the node-to-edge distance tolerance ( $\mathrm{T}=30)$ and then Our algorithm (WSNM) is compared with PEGASIS [8] and Enhanced-PEGASIS [10].

We make the following assumptions and scenarios.

- The sink locations: $(50,200),(50,300)$ and $(50,400)$.

- The number of sensor nodes: 100, 150, 200 and 250.

- Every scenario is simulated within the network field of $100 \mathrm{~m} \times 100 \mathrm{~m}$.

- The homogeneous sensor nodes are randomly distributed in the network field.

- All sensor nodes are aware of their own location (i.e. GPS signals).

- They are stationary after distribution and can send data to a sink and adjust their transmission range.

- They cannot be recharged, and an energy is restricted.

Table 1. Parameter

\begin{tabular}{|c|c|c|}
\hline Symbol & Value & Description \\
\hline Eelec & $50 \mathrm{~nJ} / \mathrm{bit}$ & The transmitter or receiver circuitry \\
\hline Camp & $100 \mathrm{pJ} / \mathrm{bit} / \mathrm{m} 2$ & The transmitter amplifier \\
\hline Eagg & $5 \mathrm{~nJ} / \mathrm{bit} / \mathrm{message}$ & The energy for data aggregation \\
\hline $\mathrm{k}$ & $2000 \mathrm{bit}$ & Message size \\
\hline Einit & $1 \mathrm{~J}$ & Initial energy of a node \\
\hline
\end{tabular}

Table 2. Radio model

\begin{tabular}{|c|c|}
\hline Radio model & $\begin{array}{l}\text { Formulas } \\
\end{array}$ \\
\hline \multirow{2}{*}{ Transmitting } & $\begin{array}{c}\text { ETX }(k, d)=\text { ETX-elec }(k)+\text { ETX-amp (k, } \\
\text { d) }\end{array}$ \\
\hline & $\operatorname{ETX}(\mathrm{k}, \mathrm{d})=$ Eelec $* \mathrm{k}+$ Camp $^{*} \mathrm{k} * \mathrm{~d} 2$ \\
\hline \multirow{2}{*}{ receiving } & ERX $(\mathrm{k})=$ ERX-elec $(\mathrm{k})$ \\
\hline & $\operatorname{ERX}(\mathrm{k})=$ Eelec $* \mathrm{k}$ \\
\hline
\end{tabular}

\subsection{Network lifetime of WSNM, PEGASIS and enhanced-PEGASIS}

In [Figure 4], (a), (b) and (c) indicate the performance of the network lifetime for the different number of sensor nodes. As we see in the overall simulation results, each algorithm shows similar results in every case irrespective of the sink location.

The simulation results are presented that WSNM shows that increase in network lifetimes of approximately 2 to 5 times more efficient than PEGASIS and Enhanced-PEGASIS. The time of the first sensor node to die in WSNM is longer than other algorithms. This means that most of the sensor nodes communicate to their nearest neighbors in WSNM. It also has a close relation to the long communication distance. 




(a) Sink location at $(50 * 200)$

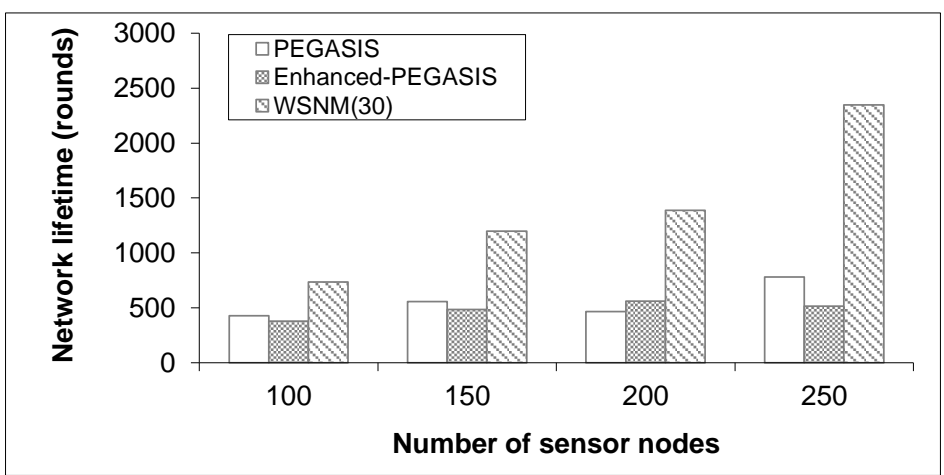

(b) Sink location at $(50 * 300)$

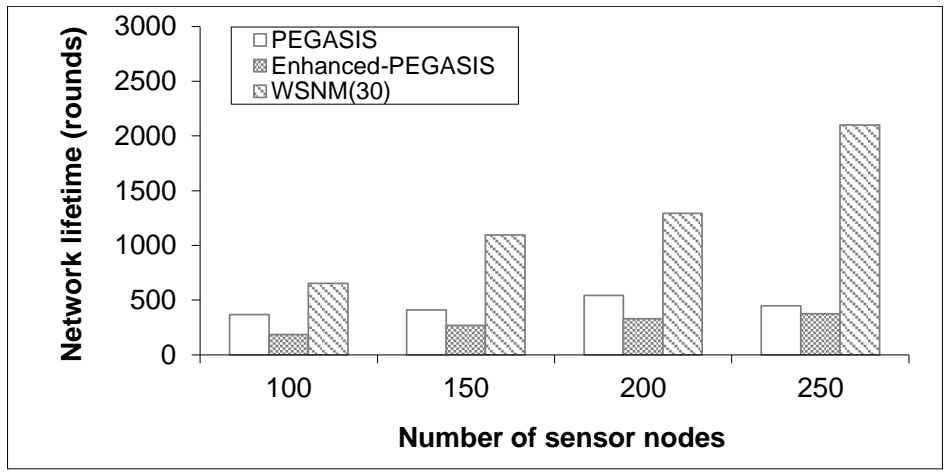

(c) Sink location at $(50 * 400)$

Figure 4. Network lifetime of three algorithms in different number of nodes

\section{Conclusions}

In this paper, we propose a wireless sensor network model (WSNM) using Douglas-Peucker algorithm in the IoT environment. The main goal of WSNM is to form a balanced chain that being able to prolong the network lifetime by improving the unbalanced energy consumption caused by the long-distance communication of some nodes in the network field. To achieve this goal, we segment the network field by using Douglas-Peucker algorithm and form a balanced chain based on small segments. We present the Network Lifetime for comparing the performance of WSNM with PEGASIS and Enhanced-PEGASIS. The simulation results show that the performance of WSNM in terms of network lifetime via a balanced energy consumption outperforms better than PEGASIS and Enhanced-PEGASIS. 


\section{Acknowledgements}

This work (Grants No.S2634356) was supported by project for Cooperative R\&D between Industry, Academy, and Research Institute funded Korea Ministry of SMEs and Startups in 2018.

\section{References}

[1] Alaba F.A., Othman M., Hashem I.A.T., and Alotaibi F., "Internet of things security: A survey," Journal Network and Computer, vol.88, pp.10-28, (2017) DOI: 10.1016/j.jnca.2017.04.002

[2] J. Shen, A. Wang, C. Wang, P. C. K. Hung, and C. F. Lai, "An efficient centroid-based routing protocol for energy management in WSN-assisted IoT," IEEE Access, vol.5, pp.18469-18479, (2017) DOI: 10.1109/ACCESS.2017.2749606

[3] D.H. Douglas and T.K. Peucker, "Algorithms for the reduction of the number of points required to represent a digitized line or its caricature," Cartographer, vol.10, no.2, pp.112-122, (1973) DOI: 10.3138/FM57-6770U75U-7727

[4] D.H. Douglas and T.K. Peucker, "Algorithms for the reduction of the number of points required to represent a digitized line or its caricature," John Wiley \& Sons, Ltd, (2011)

[5] OMNeT++, "OMNeT++, discrete event simulator," https://www.omnetpp.org

[6] K. Wehrle and M. Gunes, J. Gross, "Modeling and tools for network simulation," Springer, (2005)

[7] W.R. Heinzelman, A. Chandrakasan, and H. Balakrishnan, "Energy-efficient communication protocol for wireless microsensor networks," IEEE Proceedings of the 33rd Annual Hawaii International Conference on System Sciences, January 1-10, Maui, HI, USA, (2000) DOI: 10.1109/HICSS.2000.926982

[8] S. Lindsey and C.S. Raghavendra, "PEGASIS: power efficient gathering in sensor information systems," Proceedings of the IEEE Aerospace Conference, March, Big Sky, MT, USA, (2002) DOI: 10.1109/AERO.2002.1035242

[9] Nahdia Tabassum, A K M Ahsanul Haque, and Yoshiyori Urano, "COSEN: A chain oriented sensor network for efficient data collection," Proceedings of the Third International Conference on information Technology: New Generations, April, Las Vegas, NV, USA, (2006) DOI: 10.1109/ITNG.2006.44

[10] S. M. Jung, Y. J. Han, and T. M. Chung, "The concentric clustering scheme for efficient energy consumption in the PEGASIS," Proceedings of the 9th International conference on Advanced Communication Technology, Feb., Okamoto, Kobe, Japan, (2007) DOI: 10.1109/ICACT.2007.358351

[11] S. J. Lim, A. K. Bashir, S. Y. Rhee, and M. S. Park, "Energy based re-transmission algorithm of a leader node's neighbor node for reliable transmission in the PEGASIS," Ubiquitous Computing and Multimedia Applications, vol.150, pp.120-128, (2011) DOI: 10.1007/978-3-642-20975-8_13

[12] Lim, S.-J., G.-C. Han, G.-J. Kim, and H.-R. Park., "Efficient routing algorithm for data gathering in wireless sensor networks," Proceedings of the 3rd International Conference on Smart Media and Applications, (2014)

[13] Lim, S.-J., Gwang-Jun Kim, and Daehyon Kim., “An energy efficient clustering in Wireless Sensor Networks," Proceedings of the 3rd international Conference on Computer, Information and Application, (2015)

[14] Jin Wang, Yu Gao, Kai Wang, Arun Kumar Sangaiah, and Se-Jung Lim, “An affinity propagation based selfadaptive clustering method for wireless sensor networks," Sensors, vol.19, no.11, (2019) DOI: $10.3390 / \mathrm{s} 19112579$

[15] Jin Wang, Yu Gao, Wei Liu, Wenbing Wu, and Se-Jung Lim, “An asynchronous clustering and mobile data gathering schema based on timer mechanism in wireless sensor networks," Computers, Materials \& Continua, vol.58, no.3, pp.711-725, (2019) DOI:10.32604/cmc.2019.05450 


\section{Authors}

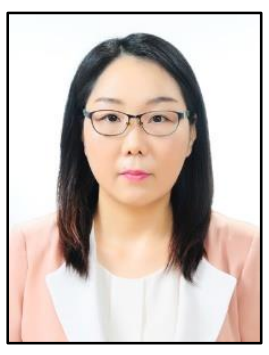

\section{Se-Jung Lim}

Graduated from the department of Computer Engineering at Chonnam National University in 2008. She received her a master's degree and doctor's degree from Chonnam National University in 2010 and in 2016. She worked for Huneed Technologies CO., LTD as a senior researcher from 2013 to 2014. In April 2019, she joined Honam University and is currently an assistant professor of Liberal Arts \& Convergence Studies Division of Convergence at Honam University. Her research interest includes Wireless Sensor Networks, Internet of Things and Big Data.

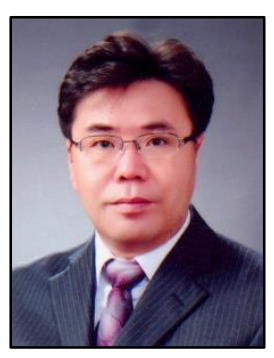

\section{Gwang-Jun Kim}

Received the B.E, M.E and Ph. D. degrees in computer engineering from Chosun University in 1993, 1995 and 2000, respectively. He joined the department of computer engineering, Chonnam National University, in 2003 and became an Associate Professor in 2009. Since 2015, he has been a Professor in computer engineering at Chonnam National University. During 2000-2001, he was a researcher in the department of electrical and computer engineering at university of California, Irvine. His current research interests focus on the area sensor network, IoT, real-time communication and various kinds of communication systems. He is a member of ISS of Korea, ICS of Korea, ITE of Korea and IMICS of Korea. 
A Wireless Sensor Network Model Using Douglas-Peucker Algorithm in The IoT Environment

This page is empty by intention. 